\title{
Emphysematous Gastritis on Computed Tomography
}

${ }^{*}$ Asma AlHatmi, ${ }^{1}$ Sameer B. Raniga, ${ }^{2}$ Alok Mittal ${ }^{2}$

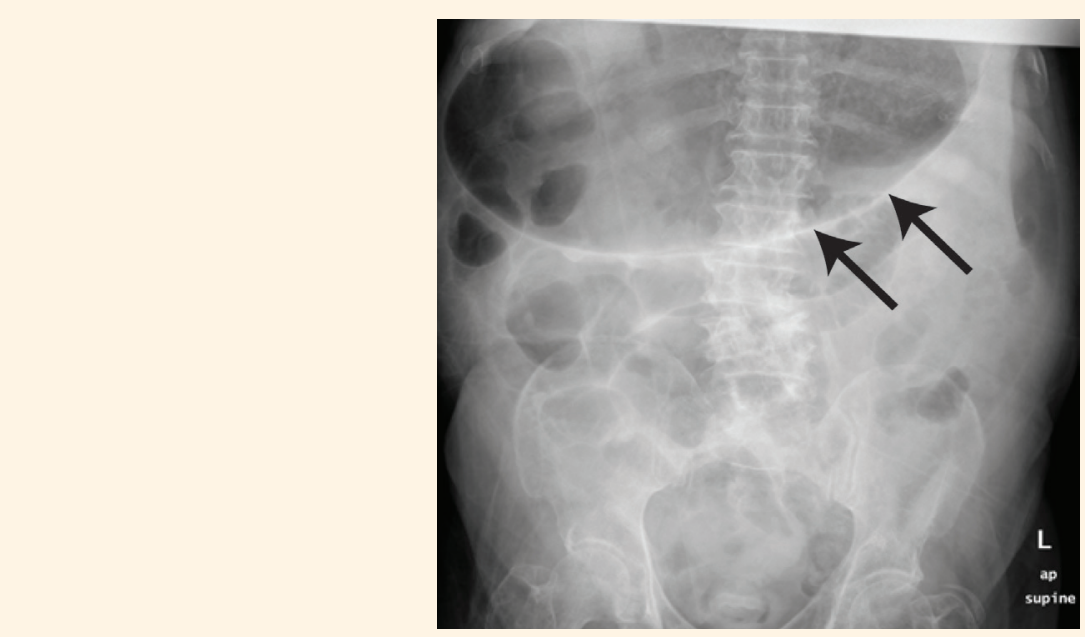

Figure 1: Plain radiograph of the abdomen of a 67-year-old male patient anteroposterior supine view showing grossly distended stomach (black arrows) without signs of pneumoperitoneum or small bowel obstruction.

\section{A} 67-YEAR-OLD OMANI MAN WITH MULTIPLE comorbidities of diabetes, hypertension, ischaemic heart disease, recurrent urosepsis and bedridden on permanent suprapubic catheterisation due to previous history of spinal trauma and surgery presented to the Emergency Department at a tertiary healthcare centre in Muscat, Oman, in 2017; he complained of acute onset of severe abdominal pain, abdominal distention and multiple episodes of vomiting for two days. The pain was localised in the epigastric region with mild localised tenderness. His vitals were normal. He had a temperature of $37^{\circ} \mathrm{C}$, pulse of 80 beats per minute, blood pressure of 125/75 $\mathrm{mmHg}$ and oxygen saturation of $98 \%$ on room air. Initial work-up showed a white blood cell count of $12,000 / \mu \mathrm{L}$ and normal lipase level. Plain radiograph of the abdomen showed a grossly distended stomach without signs of pneumoperitoneum or small bowel obstruction [Figure 1]. Computed tomography (CT) scan of the abdomen was done to exclude the possibility of gastric outlet obstruction. It showed a grossly distended stomach with mild thickened wall and extensive intramural air involving fundus, body and pyloric region [Figures $2 \mathrm{~A}$ and B]. Multiple air loculi were seen within the draining perigastric veins and within the intrahepatic portal vein in the left lobe of the liver [Figures $2 \mathrm{C}$ and $\mathrm{D}$ ].

Emergent exploratory laparotomy was done as the patient condition deteriorated; this showed a completely gangrenous stomach. A total gastrectomy was performed. The patient, who was kept in the intensive care unit and treated with sepsis protocol, later recovered. The histopathology report revealed emphysematous gastritis with acute ischaemic infarction. Literature review shows few case reports of this condition. Therefore, we report the CT findings of emphysematous gastritis associated with gastric infarction in a diabetic patient. It is the first case of emphysematous gastritis reported in the presenting healthcare centre using only radiological modality.

The reporting of the current case does not contravene with the regulations of the local institutional review board.

\section{Comment}

Emphysematous gastritis is a rare and potentially lethal condition which requires prompt diagnosis and urgent aggressive management. ${ }^{1}$ However, the presentation can be similar to other diagnoses 

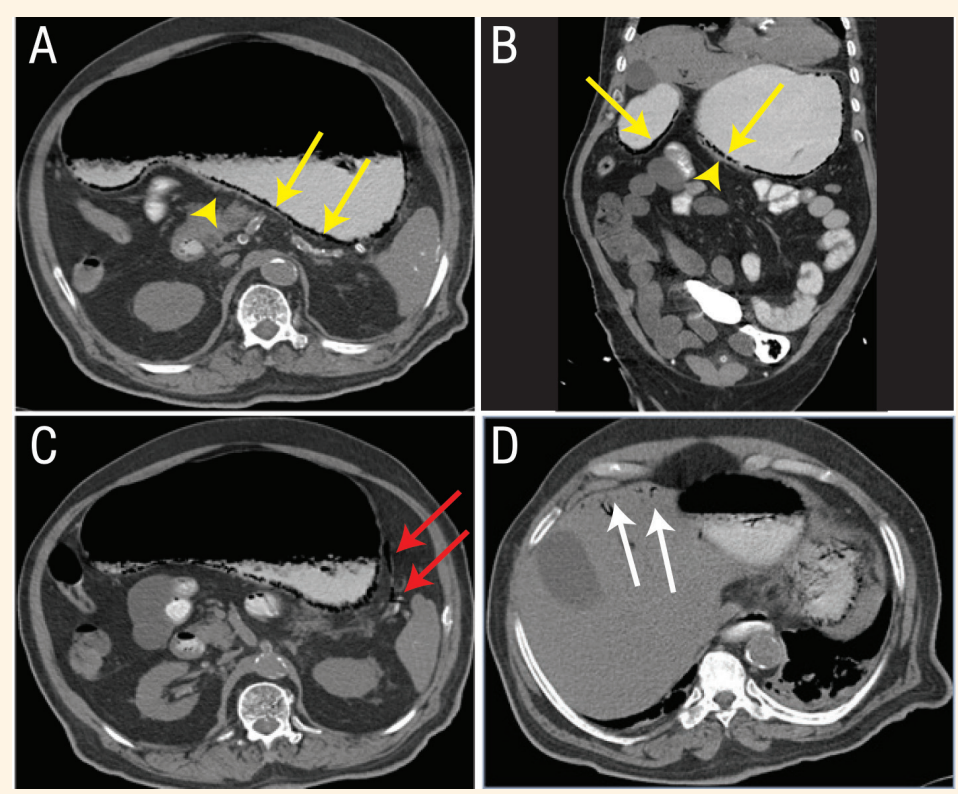

Figure 2: Non-contrast computed tomography scan of the abdomen of a 67-year-old male patient. A \& B: Axial image at upper pole of right kidney and coronal image obtained at mid abdomen level showing grossly distended stomach with tiny bubbly intramural air (arrows) and mild perigastric fatty streakiness (arrowheads). C: Axial image at mid-renal pole showing minimal air within perigastric draining vein (red arrows). D: Axial image obtained at level of gall bladder fossa showing peripheral branching linear lucencies in left lobe of liver consistent with portovenous air (white arrows).

such as gastric emphysema and gastric ischaemia. ${ }^{2}$ Differentiation is very crucial between these as the management and prognosis will also be different. A history of diabetes, ingestion of alcohol or corrosive material, adenocarcinoma of the stomach, peptic ulcer, infarction, trauma or invasion of submucosa by gasforming organism are considered to be predisposing factors to this condition. ${ }^{1,3}$ The literature shows that commonly isolated organisms are Streptococci, Escherichia coli, Enterobacter and Pseudomonas. ${ }^{3}$ The current patient had two risk factors including history of diabetes and recurrent urosepsis. Patients with emphysematous gastritis are usually symptomatic and may present with sudden acute abdominal pain, haematemesis, fever, leukocytosis or septic shock with sign of instability and systemic toxicity. ${ }^{4}$ Emphysematous gastritis is an emergency condition which needs to be considered in the differential diagnosis of acute abdomen especially in presence of risk factors and requires urgent $\mathrm{CT}$ scan of the abdomen for early diagnosis and intervention. ${ }^{5}$

Emphysematous gastritis is different from gastric emphysema in which the latter is a benign condition caused by disrupted mucosa and secondary air entry to the wall due to variable causes such as after endoscopy, extension of pneumomediastinum or pneumothorax, severe vomiting or pneumatosis cystoides. ${ }^{2}$ However, the patient remains asymptomatic and the condition will resolve spontaneously.

Both emphysematous gastritis and gastric emphysema can be manifested in plain abdominal radiograph as linear thin lucencies along the stomach wall. ${ }^{2,5} \mathrm{CT}$ is the most sensitive method and the best diagnostic modality which helps to differentiate further between these two conditions as emphysematous gastritis is usually associated with thickened gastric mucosal folds and oedema along with cystic pockets of air in the gastric wall and sometimes air in the gastric venous drainage and portal vein in severe conditions. ${ }^{2,4,5} \mathrm{CT}$ also helps to exclude other differential diagnoses of acute abdomen such as acute pancreatitis or perforated viscus. ${ }^{2}$ Plain radiograph of the abdomen of the current patient showed a grossly distended stomach without signs of pneumoperitoneum or small bowel obstruction and a CT scan was done to exclude the possibility of gastric outlet obstruction. It showed extensive intramural air involving the stomach with multiple air loculi seen within the draining perigastric and intrahepatic portal veins.

Patients with gastric emphysema are usually asymptomatic and have a benign course compared to a patient with emphysematous gastritis; most of the latter patients are clinically ill and need urgent management. ${ }^{2}$ The mortality rate from emphysematous gastritis is more than $60 \%$ and the non-lethal complication rate (e.g. gastric strictures) is up to $25 \%{ }^{1,3}$ Early nonoperative medical management with good hydration, broad-spectrum intravenous antibiotics and bowel rest improve the clinical outcomes. ${ }^{4}$ Surgery is not indicated in an acute setting even in the presence of portal venous air or pneumoperitoneum as it 
is associated with increased mortality and postoperative complications such as anastomotic leak and fistula formation. Surgery is indicated in case of clinical deterioration, peritonitis, gastric infarction, perforation or failed medical management., ${ }^{2,3}$ The current patient underwent emergent exploratory laparotomy as his condition deteriorated; this revealed a completely gangrenous stomach for which a total gastrectomy was performed. Early detection and management of emphysematous gastritis based on clinical presentation and CT findings will improve the clinical course and reduce the mortality and morbidity. ${ }^{4}$

\section{AUTHORS' CONTRIBUTIONS}

AA collected the clinical data, medical images, did the literature review, wrote the manuscript draft and edited it after review. SBR reviewed the manuscript drafts before submission for error correction. AM chose the appropriate images and wrote the captions and citations. Allauthors approved the final version od this manuscript.

\section{References}

1. Suwannachod C, Rujitanon P. A case report: Emphysematous gastritis. Int Surg J 2017; 4:2819-22. https://doi.org/10.1820 3/2349-2902.isj20173426.

2. Matsushima K, Won EJ, Tangel MR, Enomoto LM, Avella DM, Soybel DI. Emphysematous gastritis and gastric emphysema: Similar radiographic findings, distinct clinical entities. World J Surg 2015; 39:1008-17. https://doi.org/10.1007/s00268-0142882-7.

3. Shipman PJ, Drury P. Emphysematous gastritis: Case report and literature review. Australas Radiol 2001; 45:64-6. https:// doi.org/10.1046/j.1440-1673.2001.00878.x.

4. Paul M, JohnS, Menon MC, GolewaleNH, Weiss SL, MurthyUK. Successful medical management of emphysematous gastritis with concomitant portal venous air: A case report. J Med Case Rep 2010; 4:140. https://doi.org/10.1186/1752-1947-4-140.

5. Guniganti P, Bradenham CH, Raptis C, Menias CO, Mellnick VM. CT of gastric emergencies. Radiographics 2015; 23:75-87. https://doi.org/10.1148/rg.2015150062. 